\title{
Operant force-band differentiation by rats using two different response topographies
}

\author{
MICHAEL A. KIRKPATRICK and STEPHEN C. FOWLER \\ The University of Mississippi, University, Mississippi
}

\begin{abstract}
The ability of laboratory rats to differentiate the peak force of operant response was assessed for two distinct response topographies. One group of 8 rats was trained to reach through an aperture in the front panel of an operant chamber and exert downward vertical pressure on a forcesensing circular disk. A second group of 9 animals was similarly shaped to grasp and pull a wire bail attached to a force transducer located outside a second chamber. Following measurement of initial force levels during continuous reinforcement (CRF) for all responses, various force-band requirements were imposed on both groups. Only responses whose peak force amplitude fell within the band requirement (both lower and upper peak force criteria) were reinforced. Evidence for force differentiation was observed in varying degrees for both response topographies, even though the pull topography initially produced forces much higher than those emitted by the press response. The results are discussed in terms of both qualitative and quantitative changes in response characteristics, which occurred across force requirements and topographies.
\end{abstract}

Numerous studies have demonstrated that rats can, under appropriate conditions, differentiate the downward force they exert on a circular disk (Fowler, Ford, Gramling, \& Nail, 1984; Fowler, Lewis, Gramling, \& Nail, 1983; Harrell \& Fowler, 1977; Notterman \& Mintz, 1965). Even in the absence of any contingency that rewards only responses between upper and lower peak force values (a force "band"), rats responding with the downward press topography will adjust their forces to approximate the minimum force criterion for reinforcement (e.g., Fowler, Gramling, \& Liao, 1986; Notterman \& Mintz, 1965). However, in one study in which the downward press topography was compared with responding in the horizontal plane on a grasp-and-pull type manipulandum, rats using the grasp-and-pull response needlessly exerted forces three to four times greater than was necessary to procure reward (Fowler et al., 1983). The authors hypothesized that such needless overexertion may have been due to the rats' inability to differentiate the force of the grasp-and-pull response, and that this apparent lack of control may have been the result of unconditioned or elicited components of response (see also Jenkins \& Moore, 1973). The present study was designed to assess whether rats can differentiate the force of a grasp-andpull type response when confronted with appropriate contingencies.

\section{METHOD}

\section{Subjects}

The subjects were 19 male Sprague-Dawley derived rats weighing between 200 and $240 \mathrm{~g}$ upon receipt from the Holtzman Company. The

This research was supported in part by HHS Grant MH43429. Correspondence may be addressed to Stephen C. Fowler, Department of Psychology, University of Mississippi, University, MS 38677. animals were housed individually and fed ad libitum. Their body weights were kept nearly constant by restricting water access to 3-10 min daily, $30 \mathrm{~min}$ after each experimental session. In the vivarium, an automatic timer provided illumination between the hours of 8:00 a.m. and 10:30 p.m. During the course of the experiment, one animal in the press group died from an undiagnosed illness. A second animal, assigned to the grasp-and-pull group, failed to learn the task adequately and was dropped from the experiment. Data for a total of 17 animals were included in all analyses.

\section{Apparatus}

The two operant chambers used in this study have been described in detail in a previous paper (Fowler et al., 1983). Contingencies were programmed and data were recorded via a laboratory computer system, which consisted of an Apple II and a Franklin ACE 1000 microcomputer, each with associated peripherals. The system's details are provided in Fowler (1985). Each computer controlled an A/D converter that sampled the analog voltage from the transducer every $0.01 \mathrm{sec}$. From the digitized measurements were recorded the maximum force amplitude (peak-force) and duration of each response that met or surpassed a 4-g threshold. Also recorded were the response rate and the number of reinforcements obtained per session.

\section{Procedure}

Following approximately 2 weeks of exposure to restricted water intake, one group of 10 rats was shaped via the method of successive approximations to reach through the aperture in the front chamber wall and to grasp and pull a wire bail. A second group of 9 animals was similarly trained to reach through the aperture and exert downward force on a press-type manipulandum. Locating the operanda outside the chambers encouraged relatively uniform topographies and precluded the measurement of any biting or bumping of the instruments as responses.

For the first five sessions following shaping, all the animals were maintained on a CRF schedule that provided reinforcement for every response that reached or exceeded a constant 4-g threshold. Thereafter, reinforcement was provided only for responses that fell within a specified band of forces. Each group was exposed to various force-band requirements throughout the experiment, as outlined in Table 1 . The use of markedly disparate force bands for the two topographies was based on prior demonstrations that Weber's law applies to force emission in rats just as it does in humans (Fowler, 1987; Notterman \& Mintz, 1965). The first force bands imposed were positioned to include part of the distribution of forces spontaneously produced by each group of animals under the 
Table 1

Contingencies for both Press and Grasp-and-Pull Topographies, Number of Sessions, and Proportion of Reinforced Responses Under Each Force-band Requirement

\begin{tabular}{ccccc}
\hline $\begin{array}{c}\text { Force } \\
\text { Band }\end{array}$ & $\begin{array}{c}\text { Grams for } \\
\text { Band }\end{array}$ & $\begin{array}{c}\text { Sessions } \\
\text { Mann \% in Band }\end{array}$ & $\begin{array}{c}\text { Weber } \\
\text { Fraction }\end{array}$ \\
\cline { 1 - 3 } Band 1 & $20-25$ & 15 & 18.2 & .25 \\
Band 2 & $30-36$ & 8 & 20.6 & .20 \\
Band 3 & $35-42$ & 14 & 24.2 & .20 \\
Band 4 & $10-15$ & 28 & 32.7 & .50 \\
& \multicolumn{5}{c}{ Gress } \\
Band 1 & $100-125$ & 15 & 31.2 & .25 \\
Band 2 & $100-120$ & 8 & 26.4 & .20 \\
Band 3 & $100-120$ & 14 & 27.3 & .20 \\
Band 4 & $20-30$ & 28 & 20.4 & .50 \\
\hline
\end{tabular}

no-band CRF condition, and were chosen to be approximately equal in difficulty according to Weber's law (i.e., press: $5 \mathrm{~g} / 20 \mathrm{~g}=.25$; grasp-and-pull: $25 \mathrm{~g} / 100 \mathrm{~g}=.25$ ).

\section{RESULTS}

An $F$ test for the force emission prior to force-band imposition showed a difference in mean peak force between the two topographies under CRF $[F(8,7)=23.91$, $p<.0001]$. This large peak force difference between press and grasp-and-pull topographies was consistent with Fowler et al. (1983).

With respect to force-band differentiation training, a split-plot factorial analysis of variance (SPF-ANOVA) for peak force revealed significant main effects for topography $[F(1,15)=512.06, p<.00005]$, band $[F(4,60)=$ $63.39, p<.00005]$, and the topography $\times$ band interaction $[F(4,60)=22.25, p<.00005]$. Mean peak forces tended to "track" the band requirements as they changed. Note especially in Figure 1 that the 20-30-g force band (Band 4) for the grasp-and-pull topography produced a

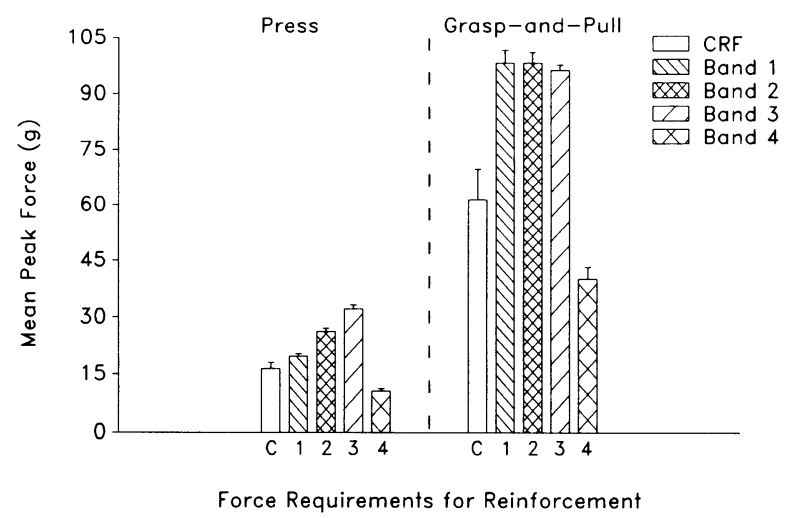

Figure 1. Mean peak force for press (first series) and pull (second series) topographies across all conditions. Vertical bars represent standard error of the mean. The upper and lower force-band requirements for each band are given in Table 1. dramatic reduction in peak force, compared with the 100-120-g band. In addition, the grasp-and-pull group continued to emit many responses with forces beyond the upper band limit, as evidenced by the mean of the peak force distributions, which lay near $\mathbf{4 0} \mathrm{g}$ for the Band 4 condition.

\section{DISCUSSION}

Initial differences between press and grasp-and-pull groups in the absence of any explicit force requirement (other than the 4-g threshold) support previous findings that the pull topography encourages unnecessarily high forces (see e.g., Fowler et al., 1983). Rats in the grasp-andpull condition initially produced forces almost six times greater than the press rats did, despite the identical 4-g threshold. When faced with appropriate contingencies, however, animals responding with either topography were able to differentiate their peak forces. The close proximity in magnitude of the force emitted to the force required supports the visually apparent "tracking"' of the requirements seen in Figure 1. Additionally, the clear reduction in force by the pull animals under the relatively low-force $20-30-g$ condition suggests that they can reduce the magnitudes of their peak forces to levels far below those observed in the absence of externally imposed force requirements. Nevertheless, the decrease in the proportion of reinforced responses for the pull group, when lower forces were required, suggests that the grasp-and-pull topography is not conducive to the same degree of control as was displayed by the press group under similar low-force conditions (see $\mathrm{Ta}$ ble 1). In contrast to the pull group, the press rats increased their proportion of reinforced responses with each new band, irrespective of the fact that the first three bands were of increasing magnitude, while the last was markedly lower. The diminished success of the pull subjects occurred despite efforts to maintain comparable task difficulty across topographies by keeping the absolute force requirement proportional to the size of the difference limen in accordance with Weber's law.

The across-topography variation in differentiation may depend upon differences in the neuromuscular involvement associated with the different planes of force emission. Alternatively, the grasping encouraged by the grasp-and-pull type operandum, but uncharacteristic with the press-type arrangement, may produce more salient proprioceptive feedback, which in turn may elicit unconditioned components of response that interfere with differentiation (Jenkins \& Moore, 1973). Such unconditioned tendencies may be related to burrowing or scratch reflexes (Fowler, Filewich, \& Leberer, 1977), or to the animals' use of flexion to draw prey or food objects to the mouth.

\section{REFERENCES}

FOWLER, S. C. (1985). Amplitude measures of operant response: Implementation with Apple Pascal. Behavior Research Methods, Instruments, \& Computers, 17, 301-306.

Fowler, S. C. (1987). Force and duration of operant response as dependent variables in behavioral pharmacology. In T. Thompson, P. B. Dews and J. E. Barrett (Eds.), Advances in Behavioral Pharmacology (Vol. 6, pp. 83-127). New York: Erlbaum.

Fowler, S. C., Filewich, R. J., \& Leberer, M. R. (1977). Withinresponse force variations accompanying duration differentiation in rats. Psychological Reports, 40, 1271-1284.

Fowler, S. C., Ford, K. E., Gramling, S. E., \& Nail, G. L. (1984). Acute and subchronic effects of neuroleptics on quantitative measures of discriminative motor control in rats. Psychopharmacology, 84, 368-373.

Fowler, S. C., Gramling, S. E., \& Liao, R. (1986). Effects of pimozide on emitted force, duration and rate of operant response maintained at low and high levels of required force. Pharmacology, Biochemistry \& Behavior, 25, 615-622.

Fowler, S. C., LewIS, R. M., Gramuing, S. E., \& NAIL, G. L. (1983). 
Chlordiazapoxide increases the force of two topographically distinct operant responses in rats. Pharmacology, Biochemistry \& Behavior, 19, 787-790.

Harrell, J. V., \& Fowler, S. C. (1977). Amount of reinforcer and differentiation of response force. Bulletin of the Psychonomic Society, 10, 358-360.

Jenkins, H. M., \& Moore, B. R. (1973). The form of the auto-shaped response with food or water reinforcers. Journal of the Experimental Analysis of Behavior, 20, 163-181.

Notterman, J. M., \& MinTZ, D. E. (1965). Dynamics of response. New York: Wiley.

(Manuscript received June 21, 1988.) 